\title{
On the Optimal Design of a Spectrum- Switched Optical Network With Multiple Modulation Formats and Rates
}

\author{
Ion Popescu, Isabella Cerutti, Nicola Sambo, and Piero Castoldi
}

\begin{abstract}
Spectrum-switched optical networks (SSONs) are an attractive solution for core networks. With respect to wavelength-switched optical networks (WSONs), they achieve a higher spectrum efficiency thanks to the use of a flexible grid, instead of a fixed one. In SSONs, the wellknown wavelength assignment (WA) problem of WSONs becomes a spectrum assignment (SA) problem. The SA problem aims at assigning a portion of the spectrum (called a frequency slot) to lightpaths. The width of the frequency slot depends on the requested bit rate and the modulation format adopted for the lightpath, and it is a multiple of the minimum bandwidth granularity, referred to as frequency slice. Thus, differently from WA, which assigns a single wavelength (or color) to each lightpath, SA assigns a set of colors (i.e., a set of frequency slices) to the lightpath. The shift from WA to SA introduces an additional constraint, which is related to the spectral adjacency of such frequency slices. This paper proves that the adjacency constraint in SA is not required and that by solving the WA problem (or the coloring problem) it is possible to derive a solution with spectrally adjacent slices in polynomial time. Based on such results, an integer linear programming formulation (ILP) for the optimal SA in a SSON with multi modulation formats and multi line rates (MMF/MLR) is presented for minimizing the network cost. The total cost of the network comprises the spectrum cost and the transponder card cost. Optimal results are presented for a MMF/MLR-SSON ring and show the amount of total occupied bandwidth and the network costs for different loads and ring lengths. Optimal selection of the modulation format and line rate is driven by the slice cost and the transponder card cost. Result comparison indicates that support of MMF and MLR is especially effective for improving spectrum utilization and allows spectrum saving up to $20 \%$ with respect to an SSON ring with single modulation format and line rate.
\end{abstract}

Index Terms-Flexible grid; ILP; Spectrum assignment; Spectrum-switched optical networks; Wavelength assignment.

\section{INTRODUCTION}

$\mathbf{O}$ ver the past few years, advances in optical transmission and switching technologies have paved the way for flexible optical networks, also called spectrum-switched optical networks (SSONs) [-ㅡㄴ] . Differently from previous

Manuscript received February 6, 2013; revised August 15, 2013; accepted September 4, 2013; published October 31, 2013 (Doc. ID 185007).

I. Popescu is with Telecom Bretagne, Brest, France.

I. Cerutti (e-mail: i.cerutti@sssup.it), N. Sambo, and P. Castoldi are with the Scuola Superiore Sant'Anna, Pisa, Italy.

http://dx.doi.org/10.1364/JOCN.5.001275 optical transport networks based on a fixed grid (e.g., with a fixed channel spacing of $50 \mathrm{GHz}$ ), SSONs adopt a flexible grid that enables optical channels with diverse bandwidth and a fine channel spacing, and thus higher spectrum efficiency. The advantage of the flexible grid is made practical by the recent development of bandwidth variable wavelength selective switches (BV-WSSs). Compared to WSSs used in wavelength-switched optical networks (WSONs), the bandwidth-variability feature makes BV-WSSs capable of switching a variable portion of the spectrum. In this way BV-WSSs can be configured to switch various optical connections (i.e., lightpath), each one requiring a different amount of bandwidth on a different portion of the spectrum. This represents a relevant innovation for improving the spectrum efficiency, as the bandwidth of the lightpath can be optimized to offer the requested bit rate for the adopted modulation format. As an example of spectrum optimization, a lightpath at $100 \mathrm{~Gb} / \mathrm{s}$ using polarization multiplexing 16 quadrature amplitude modulation (PM16QAM) requires half of the theoretical Nyquist bandwidth of $100 \mathrm{~Gb} / \mathrm{s}$ polarization multiplexing quadrature phase shift keying (PM-QPSK), i.e., $14 \mathrm{GHz}$ for PM16QAM and $28 \mathrm{GHz}$ for PM-QPSK including the overhead.

In SSONs, the portion of the spectrum occupied by a lightpath is identified by a frequency slot that is defined by the central frequency (i.e., the carrier) and the slot width (i.e., the occupied bandwidth). According to ITU-T recommendations on a flexible grid [7], the central frequency may vary at a step of $6.25 \mathrm{GHz}$, while the slot width is defined as a multiple of a minimum bandwidth granularity of $12.5 \mathrm{GHz}$. Hereafter, the minimum granularity of $12.5 \mathrm{GHz}$ is called a frequency slice. As an example, for a lightpath requiring $35 \mathrm{GHz}$ of bandwidth, the corresponding slot width would be $37.5 \mathrm{GHz}$, which consists of three spectrally adjacent slices of $12.5 \mathrm{GHz}$.

The shift of paradigm from wavelength switching to spectrum switching poses additional challenges not only at the physical layer but also at the network layer. Whereas in WSONs the provisioning of lightpaths is addressed by solving the routing and wavelength assignment problem (RWA), in SSONs the problem becomes a routing and spectrum assignment (RSA) one. The main difference is that the problem of assigning bandwidth to the lightpaths has changed from an assignment of one single color (representing a fixed amount of bandwidth) to an 
assignment of a variable number of colored frequency slices. The former problem, i.e., the wavelength assignment (WA), requires the assignment of a single wavelength or color (or multiple wavelengths when wavelength converters are available) on each link of the lightpath route, so that the same wavelength is not used by any other lightpaths passing on the same link(s). The latter problem, i.e., the spectrum assignment (SA), consists of assigning a central frequency and a slot width (i.e., a set of spectrally adjacent frequency slices) to the lightpaths from source to destination, while ensuring that the frequency slices are not reserved by any other lightpaths passing on the same $\operatorname{link}(\mathrm{s})$. In the SA problem, the adjacency constraint of the frequency slices is introduced and must be enforced. Moreover, the number of slices to be assigned depends on the bit rate of the lightpath and the modulation format selected for the transmission. Thus, the SA problem is further complicated by the fact that SSON can be enabled for supporting multi modulation format (MMF) and multi line rates (MLR).

The (R)SA problem in SSON with a flexible grid is addressed by various works and can be optimally modeled by integer linear programming (ILP) formulations [2, $\underline{8}-\underline{14}]$. The objectives of such works span from maximization of the spectrum efficiency [8-11,14] to minimization of the cost due to regenerators and transponders $[12,14]$ to minimization of the overall cost including the fiber cost [13]. Similarly, an ILP formulation is proposed in [2] for an optical network without grid (i.e., the slot width can take any value and is not necessarily a multiple of $12.5 \mathrm{GHz}$ ) supporting orthogonal frequency division multiplexing signals with different modulation formats. In all these related works, the ILP formulations impose the adjacency of the frequency slices $[\underline{5}, 6]$ assigned to each given lightpath (i.e., the slices composing the frequency slot must be contiguous in the spectrum without any interruption). The adjacency constraint is also enforced in the ILP formulation presented in [15], which aims at rearranging already established connections to reduce the spectrum fragmentation. Although correct and necessary, the enforcement of the slice adjacency adds a new constraint to the formulation, leading to a more complex problem formulation with respect to the ILP formulations for (R)WA in a WSON. Also, an additional degree of complexity arises when MLR [2,8,13] and MMF [2,12-14] are supported in SSONs (or in WSONs [16]), as the line rates and the modulation formats need to be optimally selected for each lightpath. Indeed, the selected modulation format and line rate determine the number of frequency slices to be allocated to the lightpath and thus affect the SA problem.

This paper focuses on the SA problem alone (i.e., lightpath routes are fixed and precomputed) in a static SSON supporting MMF and MLR (MMF/MLR-SSON). The main relevant contribution of the paper is the proof that the SA problem need not enforce the adjacency constraint. Indeed, after solving the weighted coloring problem [17-19] on the frequency slices (without adjacency constraint), the frequency slices of each slot can be allocated in a spectrally adjacent manner in polynomial time, with the same minimum number of slices.
Based on such a finding, the SA problem is modeled with an ILP formulation as a weighted coloring problem with joint optimization of the modulation formats and line rates for a MMF/MLR-SSON. The modulation formats and the line rates are selected to guarantee an adequate quality of transmission (QoT). A distance-adaptive approach $[\underline{1}, \underline{12}, 13]$ is exploited for selecting the best combination of modulation and rate for a given lightpath length.

In this paper, the aim of the SA is the joint optimization of the spectrum efficiency and reduction of the overall network costs. In order to derive SA performance, a unidirectional SSON ring is considered and optimally designed. Optimal results of the ILP formulation aim at better understanding of the impact of the selection of modulation formats and line rates on the spectrum utilization and the network cost. For this purpose, a parametric study of the network cost using state-of-the-art modulation formats and line rates is carried out.

\section{Spectrum Assignment Problem With Fixed Modulation Format and Line Rate}

In this section, some important properties of the SA problem are derived for the case in which the modulation format and the line rate of each lightpath are fixed $a$ priori. The SA problem is defined on a SSON in which a set of lightpath demands and their corresponding routes are already given. The SA problem is defined as the problem of assigning the frequency slices, or simply slices, to each lightpath such that the continuity constraint, the conflict constraint, and the adjacency constraint are ensured on the slices [8]. The continuity constraint imposes that the same slice is assigned on each link of the lightpath route, i.e., wavelength (or slice) conversion is not allowed. The conflict constraint ensures that any two lightpaths that are sharing the same physical link are assigned to different slices. The adjacency constraint forces the frequency slices for the same lightpath to be contiguous, i.e., to be assigned spectrally contiguous colors. In summary, the SA aims at minimizing the number of assigned frequency slices while satisfying continuity, conflict, and adjacency constraints on the slices.

The SA problem is similar to the WA problem, with the additional constraint of the slice adjacency constraint. To clarify the difference, consider first the WA problem in a SMF/SLR-SSON. It is well known that the WA can be mapped to the coloring problem [20], which aims at coloring the nodes of a graph such that adjacent nodes are assigned different colors. The WA problem can thus be solved by following these steps [20]:

- Construct an auxiliary graph $\mathcal{G}(\mathcal{V}, \mathcal{E})$ such that each lightpath in the system is represented by a node $e$ in graph $\mathcal{G}$. There is an undirected edge $v$ between two nodes in graph $\mathcal{G}$ if the corresponding lightpaths pass through a common physical fiber link. This graph is named the path conflict graph. 
- Solve the coloring problem by coloring the nodes of the path conflict graph $\mathcal{G}$ such that no two adjacent nodes have the same color.

For the SA problem, it is possible to follow the same solution approach of WA. However, in the SA problem each lightpath is assigned multiple slices, instead of a single wavelength. Therefore each node of the path conflict graph has a weight indicating the number of colors (i.e., slices) to be assigned to the corresponding lightpath.

An example is presented in Fig. 1. Consider a unidirectional ring that consists of seven nodes as shown in Fig. 1 (a). The demand set $D$ is $(1,2),(1,3),(3,6),(4,7)$, and $(5,1)$, and the lightpath demands require $4,5,2,3$, and 3 frequency slices, respectively, as shown in Fig. 1(b). The routing of the lightpath demands are (1-2), (1-2-3), (3-4-5-6), (4-5-6-7), and (5-6-7-1), respectively. The graph in Fig. 1(b) represents the path conflict graph, and the weight of each node represents the required number of frequency slices per lightpath.

The problem of assigning a set of $w(v)$ colors to each node $e$, such that the colors assigned to adjacent nodes are distinct, is known as the weighted coloring problem [18]. The objective is to minimize the number of colors to be assigned. However, the SA problem includes the additional adjacency constraint, which forces the colors assigned to the same node to be contiguous. The open issue is whether an optimal solution to the weighted coloring problem can also satisfy the adjacency constraint.

To simplify the treatment of the issue on the adjacency constraint, the path conflict graph is expanded as follows. The expanded path conflict graph $\mathcal{G}_{e}\left(\mathcal{V}_{e}, \mathcal{E}_{e}\right)$ is a path conflict graph in which each node represents the requirement of a frequency slice and $w(v)$ nodes are aggregated in a super-node representing the slot of a lightpath demand. Links between nodes of the same super-node ensure that a number of colors equal to the number of contained nodes is assigned to each super-node. Links between nodes belonging to distinct super-nodes ensure that the same colors cannot be used by lightpaths passing on the same physical link.

The expanded path conflict graph for the example in Fig. 1 is shown in Fig. 2. The node IDs in the expanded path

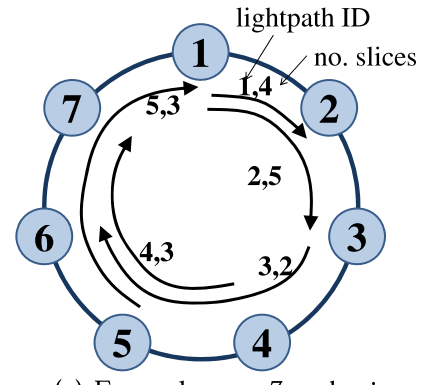

(a) Example on a 7 node ring.

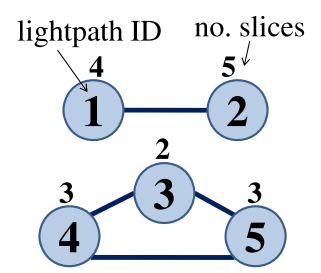

(b) Path conflict graph $\mathcal{G}(\mathcal{V}, \mathcal{E})$.
Fig. 1. Mapping the SA problem to the coloring problem for $D=\{(1,2),(1,3),(3,6),(4,7),(5,6)\}$ requiring $4,5,2,3$, and 3 slices, respectively.

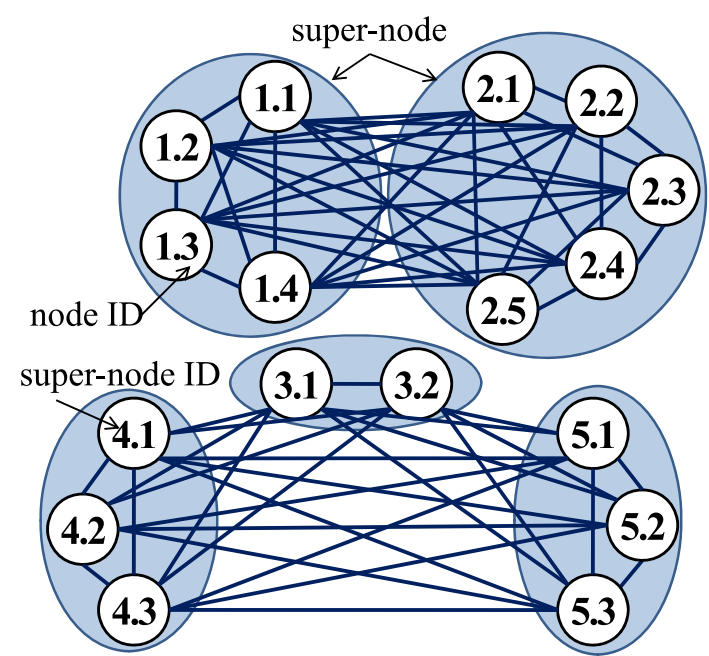

Fig. 2. Expanded path conflict graph $\mathcal{G}_{e}\left(\mathcal{V}_{e}, \mathcal{E}_{e}\right)$ for the example in Fig. 1.

conflict graph are identified as $x \cdot y$, where $x$ is the lightpath ID (or super-node ID) and $y$ is the slice ID in the original path conflict graph. For instance, node 1 of the path conflict graph in Fig. 1(b) is expanded in four nodes, linked among them and representing the frequency slices to be assigned to lightpath 1 . The links ensure that the colors to be assigned to all the nodes of a super-node (i.e., for the same lightpath) are different. In addition the link $(1,2)$ in the graph in Fig. 1(b) is replaced with a link (1.t, 2.z) between each pair of nodes $t$ and $z$ of super-nodes 1 and 2 . Such links ensure that lightpaths using the same physical links are assigned disjoint slices.

Solving the coloring problem on the expanded path conflict graph ensures the continuity and conflict constraints, i.e., that a set of (distinct) slices is assigned to each lightpath and not used by any other lightpaths passing on the same physical link. However, it is also necessary to assign contiguous (i.e., spectrally adjacent) colors to the nodes belonging to the same super-node. Next, from the properties of the expanded path conflict graph, we prove the equivalence of the SA problem and the weighted coloring problem.

\section{A. Mapping the SA Problem}

Let us first review some definitions of graph theory [18]. A clique is a subset of vertices that are all adjacent to each other. A maximum clique is the clique of largest size in a graph. When nodes are weighted (e.g., in the path conflict graph), then the weighted maximum clique is the clique whose sum of node weights is the highest.

Property 1: The maximum clique of an expanded path conflict graph contains all the nodes of an integer number of super-nodes.

Since all the nodes of a super-node are completely connected in the expanded path conflict graph, then either they all belong to the maximum clique or not. 
Let us introduce some novel definitions. Let the color ID be a positive integer assigned sequentially to the slices based on their spectral position. Let us also define the spectrum gap as the absolute value of the difference between two color IDs. In other words, the spectrum gap between colors $c_{1}$ and $c_{2}$ is $\left|c_{1}-c_{2}\right|$. Thus, when the spectrum gap is 1 , the colors are spectrally contiguous. The maximum spectrum gap of a set of colors $C=\left\{c_{1}, c_{2}, \ldots, c_{|C|}\right\}$ is given by the $\left|c_{\max }-c_{\min }\right|$, where $c_{\max }=\max _{i: c_{i} \in C} c_{i}$ and $c_{\min }=$ $\min _{i: c_{i} \in C} c_{i}$.

Theorem 1: Given an optimal solution of the coloring problem on an expanded path conflict graph consisting of $k$ (contiguous) colors, it is always possible to color the nodes of such a graph with color IDs in $\{1,2, \ldots, k\}$ in such a way that the adjacency constraint is ensured.

Proof: For a given optimal coloring on the expanded path conflict graph, it is possible to find the maximum clique (i.e., the group of nodes completely connected that are using exactly $k$ colors) in polynomial time by visiting each node and checking whether it has at least $k-1$ neighbors using $k-1$ distinct colors [21,22]. Due to Property 1, the maximum clique includes all the nodes of a single or multiple super-nodes. Once the maximum clique(s) have been found, the colors are assigned again to ensure the adjacency constraint. The adjacency constraint is ensured when the maximum spectrum gap between the colors assigned to a super-node is equal to the number of nodes in the super-node. The assignment of spectrally contiguous colors to the nodes of each super-node can be performed as follows:

- Start to assign incremental color IDs (starting from 1 and increasing by 1) to the nodes of a super-node in the maximum clique. Within such a super-node the maximum spectrum gap is equal to the number of nodes.

- Repeat the assignment operation for the other supernodes of the maximum clique by always incrementing the color ID. At the end of this step, the nodes in the maximum clique are assigned $k$ distinct contiguous color IDs.

- Color the remaining nodes as follows:

- If there are no other maximum cliques, then it is always possible to color the other remaining nodes in the super-nodes with less than $k$ contiguous color IDs.

- If the expanded path conflict graph contains other maximum cliques (i.e., if there are more maximum cliques with the same number of nodes), then the super-nodes adjacent to the colored maximum clique must reuse the color IDs previously assigned to the colored nonadjacent super-nodes. In other words, the nodes in the colored maximum clique are $k$, out of which $m$ are not adjacent to the other maximum clique to be colored. Thus, such $m$ colors can be reused and are sufficient for the adjacent nodes in the maximum clique to be colored. Indeed, the number of adjacent nodes to be colored cannot be greater than $m$; otherwise the maximum clique should be different and greater than $k$, i.e., $m<k$. Thus, it is always possible to use the $m<k$ color IDs that are contiguous. The remaining super-nodes in the maximum clique can be colored with the other $(k-m)$ contiguous colors.

- This process can then be repeated by reusing the $k$ colors while reassigning contiguous colors for the nodes in the same super-node.

By starting from the solution of the coloring problem on the expanded path conflict graph and by following these steps, it is possible to derive-in polynomial time-a color assignment satisfying the adjacency constraint and using the same number of colors. The next lemma proves that the coloring problem can be solved directly on the path conflict graph, without the need for expanding the graph.

Lemma 1: The coloring problem on the expanded path conflict graph is equivalent to the weighted coloring problem on the path conflict graph.

In both graphs, adjacent nodes must be assigned distinct colors. Also the number of colors to be assigned to a node of the path conflict graph is equal to the number of nodes in a super-node in the expanded path conflict graph. This means that the weighted coloring problem on the path conflict graph is equivalent to the coloring problem on the expanded path conflict graph.

Property 2: The minimum number of colors of the SA is equal to the weighted maximum clique size of the path conflict graph or equivalently the maximum clique size of the expanded path conflict graph.

This property derives from the fact that SA is equivalent to the weighted coloring problem and that the minimum number of colors is given by the (weighted) maximum clique size in the (weighted) coloring problem defined on the expanded path conflict graph (path conflict graph).

\section{Complexity: The SA problem is NP-complete.}

The proof of Theorem 1 showed that in polynomial time it is possible to reduce the SA problem into a weighted coloring problem, which is a NP-complete problem [18]. Thus, the SA problem is also NP-complete. The SA problem remains NP-complete even for weights $w(v)$ equal to 1 for each node $v$ : in this case the SA problem is reduced to a coloring problem that is also NP-complete.

In summary, the SA problem can be solved as a weighted coloring problem on the path conflict graph and then by assigning the contiguous color IDs. Next the ILP formulation for the SA is presented when selection of the number of slices (i.e., selection of the modulation formats and line rates) is performed jointly in a MMF/MLR-SSON. The formulation is based on the ILP formulation for the weighted coloring problem on the path conflict graph.

\section{Minimum Cost MMF/MLR-SSON With QoT CONSTRAINTS}

In this section, the SA problem is formulated in a SSON supporting MMF/MLR in an adaptive way. The SA problem is jointly solved with the selection of the modulation format(s) and line rate(s), referred to as the modulation-rate pair. To ensure QoT, the length of each 
lightpath demand is constrained to not exceed the maximum transparent reach, which is defined as the maximum propagation distance of the optical signal (without optoelectro-optical regeneration) that achieves a bit error rate lower than a given threshold. The maximum transparent reach depends on the selected modulation format and line rate. When selecting the modulation-rate pair for the lightpaths, a trade-off between maximum transparent reach and bandwidth requirement exists. Indeed, for a given line rate, increasing the spectral efficiency of the modulation format leads to a reduction of the maximum transparent reach [1].

The objective of the optimization problem is minimization of the total network cost. The total network cost is given by the spectrum cost and the transponder cost. The objective function leads to a trade-off between the spectrum utilization and the transponder cost required for a given modulation-rate pair. In general, the more spectrumefficient the modulation-rate pair, the higher the complexity of the transponder, leading to a higher cost.

\section{A. Example of the Selection of the Modulation-Rate Pair}

For an example, let us consider a set of modulation-rate pairs, their corresponding requirements of slices, and their achieved maximum transparent reach. Various examples of such set values are available in [23-26] for different transmission techniques (e.g., single carrier [23,25] or orthogonal frequency division multiplexing $[24,26])$, optical technologies, and physical layer parameters and settings (e.g., bandwidth versus reach trade-off). In this paper, the considered set of modulation-rate pairs, the number of slices, and the maximum transparent reach are taken from the experimental assessment described in $[\underline{3}, \underline{27}]$. The values are summarized in Table I. Transmission rates are expressed considering 100 and $200 \mathrm{~Gb} / \mathrm{s}$ information rates plus overhead, leading to 112 and $224 \mathrm{~Gb} / \mathrm{s}$ rates.
Hereafter, for simplicity, the line rates will be indicated as 100 and $200 \mathrm{~Gb} / \mathrm{s}$. Modulation formats are PM-16QAM and PM-QPSK. The maximum transparent reach is experimentally derived and is in the order of other experiments with similar physical layer settings, e.g., [25]. In Table I, the number of cards requested for the considered modulation-rate pairs is derived based on the transponder architecture in [27]. Such transponder architecture is composed of electronic cards (i.e., traffic tributaries) that modulate the optical carrier. A PM-16QAM transponder consists of eight cards, whereas a PM-QPSK transponder consists of four cards. As an example, a PM-16QAM transponder with eight cards at $28 \mathrm{~Gb} / \mathrm{s}$ transmits an optical signal modulated at $224 \mathrm{~Gb} / \mathrm{s}(8 \times 28 \mathrm{~Gb} / \mathrm{s})$. To support a bandwidth of $28 \mathrm{GHz}$, three slices of $12.5 \mathrm{GHz}$ are required (i.e., $3 \times 12.5=37.5>28 \mathrm{GHz}$ ).

The cost of the transponder is assumed to be proportional to the number of cards and their transmission rate. More specifically, assume that the cost increases less than the transmission rate $[8,16]$. The trade-off between the cost of the spectrum and of cards on the network design is now clarified with an example. Consider the topology and the lightpath demands in Fig. 1(a). Assume that the link lengths are equal to $d=120 \mathrm{~km}$, and assume that the lightpath demands are for $200 \mathrm{~Gb} / \mathrm{s}$ traffic. The optimal modulation-rate pair for the lightpath demands in a MMF SSON supporting either SLR at $200 \mathrm{~Gb} / \mathrm{s}$ or MLR at 100 and $200 \mathrm{~Gb} / \mathrm{s}$ is as shown in Table II. The optimal modulation format is selected according to the maximum transparent reach and the optimization objective, i.e., the minimization of either the spectrum utilization or the transponder costs in the table. Also, when MLR is enabled, it is more spectrally efficient to support each one of the lightpath demands 3-5 on two lightpaths at $100 \mathrm{~Gb} / \mathrm{s}$.

The joint problem of selecting the modulation-rate pair and performing the SA with the objective of minimizing the cost due to spectrum utilization and cards is modeled with an ILP formulation next.

TABLE I

Parameters for the Different Modulation-Rate Pairs, Considering 100 and 200 Gb/s With Overhead

Modulation-Rate Pair

\begin{tabular}{|c|c|c|c|c|c|c|}
\hline Mod. Format & Line Rate $[\mathrm{Gb} / \mathrm{s}]$ & Bandwidth [GHz] & No. of Slices & Max. Transp. Reach [km] & No. of Cards & Card bit Rate $[\mathrm{Gb} / \mathrm{s}]$ \\
\hline PM-16QAM & 112 & 14 & 2 & 400 & 8 & 14 \\
\hline PM-16QAM & 224 & 28 & 3 & 250 & 8 & 28 \\
\hline PM-QPSK & 112 & 28 & 3 & 2500 & 4 & 28 \\
\hline PM-QPSK & 224 & 56 & 5 & 2500 & 4 & 56 \\
\hline
\end{tabular}

TABLE II

Optimal Modulation-Rate Pair, for Lightpath Demands as in Fig. 1(a) and Link Lengths of $d=120 \mathrm{~km}$

\begin{tabular}{|c|c|c|c|c|c|}
\hline \multirow[b]{2}{*}{ Line Rates $[\mathrm{Gb} / \mathrm{s}]$} & \multirow{2}{*}{$\frac{\text { Lightpath }}{\text { ID }}$} & \multicolumn{2}{|c|}{ Min. Spectrum Utilization } & \multicolumn{2}{|c|}{ Min. Transponder Cost } \\
\hline & & Mod. & Rate $[\mathrm{Gb} / \mathrm{s}]$ & Mod. & Rate $[\mathrm{Gb} / \mathrm{s}]$ \\
\hline SLR (200) & $1-5$ & PM-16QAM & 200 & PM-QPSK & 200 \\
\hline $\operatorname{MLR}(100,200)$ & $1-2$ & PM-16QAM & 200 & PM-QPSK & 200 \\
\hline & $3-5$ & PM-16QAM & 100 & PM-QPSK & 200 \\
\hline
\end{tabular}




\section{B. ILP Formulation}

The path conflict graph $\mathcal{G}(\mathcal{V}, \mathcal{E})$ derived as in Section II is used by the ILP along with the following parameters and variables:

Parameters:

- $\mathcal{V}$ : set of lightpath demands

- $T_{v}$ : requested bit rate for the lightpath demand $v \in \mathcal{V}$

- $L_{v}$ : length of the lightpath $v \in \mathcal{V}$

- $M$ : set of modulation-rate pairs

- $m$ : modulation-rate pair in $M$

- $B_{m}$ : bit rate of the modulation-rate pair $m \in M$

- $S_{m}$ : number of frequency slices required for the modulation-rate pair $m \in M$

- $\chi$ : set of available frequency slices such that $|\chi| \leq\left|\mathcal{V} \cdot \sum_{m \in M} S_{m}\right|$

- $K_{m}$ : number of transponder cards required for the modulation-rate pair $m \in M$

- $H_{m}$ : maximum transparent reach for the modulationrate pair $m \in M$

- $C S$ : cost of a frequency slice

- $C C_{m}$ : transponder card cost for the modulation-rate pair $m \in M$

\section{Variables:}

- $y_{j}$ : binary variable that takes value 1 if frequency slice $j \in$ $\chi$ is used

- $x_{v j m}$ : binary variable that takes value 1 if lightpath $v \in \mathcal{V}$ is assigned to frequency slice $j \in \chi$ and belongs to the modulation-rate pair $m \in M$

- $z_{v m}$ : integer variable that indicates how many lightpaths are established for demand $v \in \mathcal{V}$ and modulation-rate pair $m \in M$ (i.e., traffic of a demand is split on multiple parallel lightpaths)

\section{Objective Function:}

$$
\min : \sum_{j \in \chi} C S \cdot y_{j}+\sum_{j \in \chi} \sum_{v \in \mathcal{V}} \sum_{m \in M} C C_{m} \cdot x_{v j m} \cdot \frac{K_{m}}{S_{m}},
$$

Subject to:

$$
\begin{gathered}
\sum_{m \in M: L_{v} \leq H_{m}} B_{m} \cdot z_{v m}=T_{v} \quad \forall v \in \mathcal{V}, \\
S_{m} \cdot z_{v m}=\sum_{j \in \chi} x_{v j m} \quad \forall v \in \mathcal{V}, \forall m \in M, \\
\sum_{m \in M} x_{v j m}+x_{v^{\prime} j m} \leq y_{j} \quad \forall\left(v, v^{\prime}\right) \in \mathcal{E}, \quad \forall j \in \chi .
\end{gathered}
$$

The objective function (1) aims to minimize the total cost of the network, which includes spectrum cost (as a function of the frequency slices) and transponder card cost. The constraint (2) assigns the modulation-rate pair to each lightpath demand, while ensuring the QoT constraint. The constraint ( $\underline{3})$ assigns a number of slices sufficient for the selected modulation-rate pair for each lightpath demand. The constraint (4) is the conflict constraint: it forces two adjacent vertices of the path conflict graph (i.e., two lightpaths routed on one or more common links of the physical topology) to be assigned different frequency slices.

Thanks to the results of Theorem 1, the adjacency constraint need not be enforced in the ILP formulation. This permits us to save a number of constraints in the order of $O(|\chi| \cdot|T| \cdot|M|)$ with respect to the previously proposed ILP formulations $[8,9,11,12]$. Note that such complexity reduction is also attainable in other ILP formulations (e.g., in $[8,9,11,12])$ and in general in any problem of RSA in SSON with mesh topologies.

\section{Optimal Results}

The proposed ILP formulation is implemented in AMPL [28] and optimally solved with the commercial solver IBM ILOG CPLEX v.12.2 [29]. To enable SA assessment, a unidirectional ring with seven nodes as illustrated in Fig. 1(a) is considered. The links have the same length of $d \mathrm{~km}$. Each node is a BV-WSS equipped with bandwidth variable transponders supporting PM-QPSK and PM-16QAM modulation formats at 100 and $200 \mathrm{~Gb} / \mathrm{s}$, as indicated in Table I. The flexible grid defined in [7] with $12.5 \mathrm{GHz}$ spacing is assumed.

First, optimal results on the spectrum utilization are collected by setting the card cost to zero in the objective function (1) of the ILP formulation. Then, optimal results on the cost of the SSON ring are presented.

\section{A. Minimizing the Spectrum Utilization}

This subsection aims to assess the impact of the selection of the modulation format and line rate on the spectrum utilization. The spectrum utilization is defined as the difference between the highest and the lowest frequencies utilized in the ring. Designs at MMF, single modulation formats (SMF), MLR, and single line rate (SLR) are compared for varying link length $d$ and loads. In the case of SMF SSON, the chosen modulation format is driven by the longest lightpath in the network; that is, the chosen modulation format is the most spectrally efficient one that ensures a transparent reach for the longest requested lightpath.

Figure 3 shows the spectrum utilization versus the link length for SLR design. The demand matrix is assumed to be uniform and complete, with the demand of a single lightpath between each node pair supporting traffic of either 100 or $200 \mathrm{~Gb} / \mathrm{s}$. A MMF design is spectrally advantageous with respect to a SMF design only when the spectrally efficient modulation formats (i.e., PM-16QAM in the considered scenario) can be used for selected lightpaths. The maximum spectrum saving is $14 \%$ at $100 \mathrm{~Gb} / \mathrm{s}$ and $10 \%$ at $200 \mathrm{~Gb} / \mathrm{s}$. Instead, when link lengths are short, the more spectrally efficient modulation formats can be used for all 


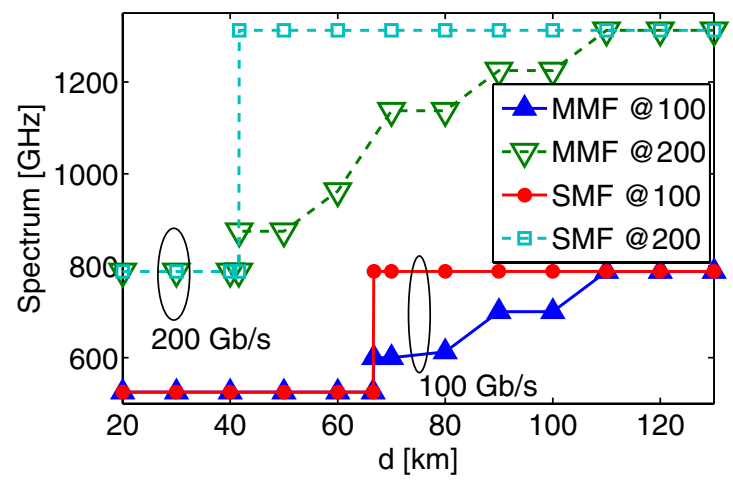

Fig. 3. Spectrum utilization versus link length $(d)$ for SLR design when the demand matrix is uniform and complete.

the lightpaths as the maximum transparency reach of such modulation formats is satisfied. Thus, there is no spectral saving in using MMF. On the other hand, for long link lengths, the maximum transparency reach can be satisfied only by the less spectrally efficient modulation formats (i.e., PM-QPSK in the considered scenario), making the spectrum utilization of SMF and MMF equal. Note that increasing the link length $(d)$ has the same effect as decreasing the maximum transparent reach for the given link lengths. In other words, Fig. 3 indicates how the spectrum utilization can improve when technological advances in optical transmission allow longer transparent reach (i.e., when decreasing $d$ ).

In Fig. 4, the spectrum utilization is presented as a function of the load (in Tb/s) supported by the ring. A number of lightpath demands are randomly generated with uniformly distributed source and destination nodes until reaching a given network load. A number of demand matrices are generated until the collected results achieve a confidence interval of $5 \%$ or better at $95 \%$ confidence level. Different link lengths, $d$, are considered in a SLR ring operating at either 100 or $200 \mathrm{~Gb} / \mathrm{s}$. The increase of bandwidth is linear with load, but with a different slope. For a network with longer link lengths, larger bandwidth is necessary, because longer lightpaths are forced to use less efficient modulation formats. When considering $d=90 \mathrm{~km}$, for a $100 \mathrm{~Gb} / \mathrm{s}$ line

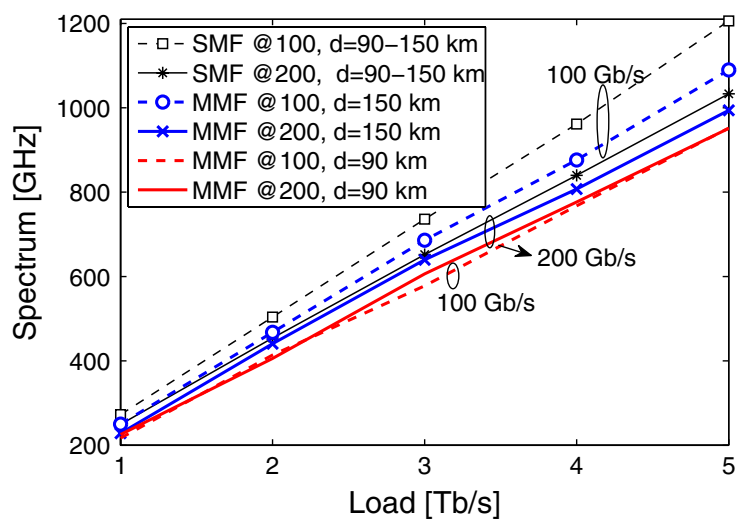

Fig. 4. Spectrum utilization versus load for randomly generated traffic demands and for different link lengths $(d)$ with SLR designs. rate, the predominant modulation format selected by the optimal solutions is PM-16QAM, whereas a mix of PM16QAM and PM-QPSK is selected for a $200 \mathrm{~Gb} / \mathrm{s}$ line rate. Thus, the average number of slices per b/s is approximately equal, leading to similar spectrum utilization at 100 and $200 \mathrm{~Gb} / \mathrm{s}$. Instead, for $d=150 \mathrm{~km}$, there is a difference in the spectrum utilization at 100 and $200 \mathrm{~Gb} / \mathrm{s}$ line rates. This is due to the fact that for both rates the predominant modulation format is PM-QPSK, and therefore to serve the same load for $200 \mathrm{~Gb} / \mathrm{s}$ it requires fewer slices per b/s compared to the $100 \mathrm{~Gb} / \mathrm{s}$ line rate (i.e., five slices per $200 \mathrm{~Gb} / \mathrm{s}$ instead of three slices per $100 \mathrm{~Gb} / \mathrm{s}$ ). If the link length is further increased, most of the lightpaths use PM-QPSK as the modulation format; thus the difference in utilization persists. The figure also evidences that the spectrum saving of MMF with respect to SMF is about $20 \%$ for a $100 \mathrm{~Gb} / \mathrm{s}$ line rate and up to $10 \%$ for a $200 \mathrm{~Gb} / \mathrm{s}$ line rate. Such spectrum saving is higher for small ring sizes (i.e., for shorter link lengths) as more spectrally efficient modulation formats can be exploited.

Figure 5 compares the spectrum utilization when enabling MMF and MLR in a ring with link lengths of $d=90 \mathrm{~km}$ and for random traffic demands of $200 \mathrm{~Gb} / \mathrm{s}$. When MLR is enabled, the lightpath demands at $200 \mathrm{~Gb} / \mathrm{s}$ can be split on two parallel lightpaths at $100 \mathrm{~Gb} / \mathrm{s}$. As an example, with MLR, for a path length between 250 and $400 \mathrm{~km}, 200 \mathrm{~Gb} / \mathrm{s}$ can be supported with two $100 \mathrm{~Gb} / \mathrm{s}$ PM-16QAM lightpaths occupying four slices each, instead of a $200 \mathrm{~Gb} / \mathrm{s}$ PM-QPSK, which occupies five slices. The comparison shows that the support of MMF allows a spectrum saving of up to $10 \%$. When also enabling MLR support, an additional spectrum saving of 5\%-10\% can be achieved. Thus, the overall spectrum saving of MMF/ MLR with respect to a SMF/SLR design can range between $14 \%$ and $19 \%$.

\section{B. Minimizing the Total Cost}

In this subsection, the total network cost of a SSON ring based on MMF is assessed as a function of the maximum load (in $\mathrm{Tb} / \mathrm{s}$ ) supported by the ring. The link lengths are fixed to $d=90 \mathrm{~km}$. For a given network load, matrices of lightpath demands at $200 \mathrm{~Gb} / \mathrm{s}$ are randomly generated

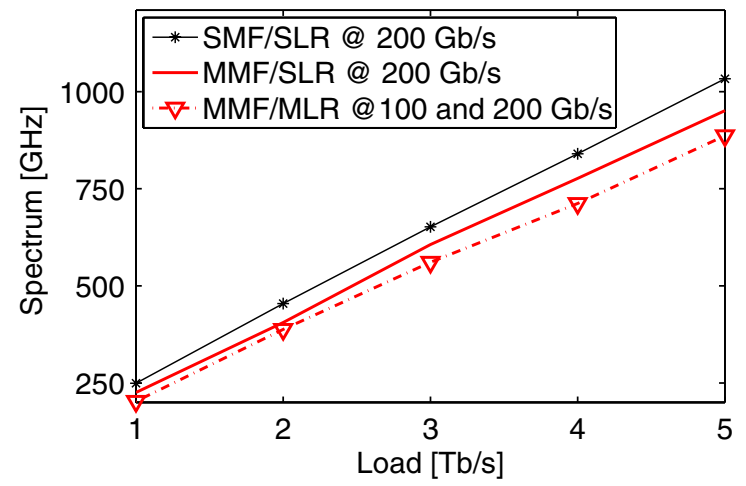

Fig. 5. Spectrum utilization versus load for randomly generated traffic demands at $200 \mathrm{~Gb} / \mathrm{s}$ and $d=90 \mathrm{~km}$. 
with uniformly distributed source and destination nodes until reaching the load of $3 \mathrm{~Tb} / \mathrm{s}$. A number of demand matrices are generated until the collected results achieve a confidence interval of $5 \%$ or better at $95 \%$ confidence level.

It is assumed that the cost increase is proportional to the transmission rate $[\underline{8}, \underline{16}]$. This assumption was valid in SONET/SDH networks in which a four-fold increase of the transmission rate of a transponder card causes approximately a doubling of the card cost. To keep the rate-cost relationship more general, it is assumed that a doubling of the transmission rate leads to an $\alpha$-fold increase of the card cost with $\alpha<2$ (e.g., $\alpha=\sqrt{2}$ in SONET/SDH networks). Thus, the cost of a single card for the modulationrate pairs in Table $\mathrm{I}$ is $C C_{1}$ (for $100 \mathrm{~Gb} / \mathrm{s}$ PM-16QAM), $C C_{2}=C C_{3}=\alpha \cdot C C_{1} \quad$ (for $200 \mathrm{~Gb} / \mathrm{s} \quad$ PM-16QAM and $100 \mathrm{~Gb} / \mathrm{s}$ PM-QPSK), and $C C_{4}=\alpha^{2} \cdot C C_{1}$ (for $200 \mathrm{~Gb} / \mathrm{s}$ PM-QPSK). Results are presented as a function of the ratio $(\gamma)$ between card cost at the lowest modulation-rate pair (the first row $m=1$ in Table I) and spectrum cost, i.e., $\gamma=C C_{1} / C S$. For simplicity, the cost of a frequency slice is normalized to 1 , i.e., $C S=1$.

Figure 6 illustrates the total cost of the network versus the ratio between the cost of the card and the cost of the frequency slice, $\gamma$, for $\alpha=1.1$ and $\alpha=1.4$, in a MMF/ SLR-SSON ring. The cost for the used spectrum remains almost constant with $\gamma$ and is equal to the number of frequency slices necessary to support the lightpath demands. Instead the cost for transponder cards (or total card cost) and the total cost of the network increase linearly with $\gamma$. The value of $\alpha$ drives the slope of the card cost and total cost: a higher value of $\alpha$ (e.g., $\alpha=1.4$ ) results in a higher cost increase with $\gamma$.

The trade-off discussed with the example in Subsection III.A is, however, better displayed in Fig. 7, which shows the average number of transponder cards and frequency slices versus $\gamma$. When $\gamma$ is equal to zero, the cost of the transponder cards is negligible with respect to the slice cost and the number of transponder cards does not influence the objective function. Thus, in the optimal design a high number of transponder cards are allocated. When increasing $\gamma$, there is a trade-off between the average number of transponder cards and the average number of frequency

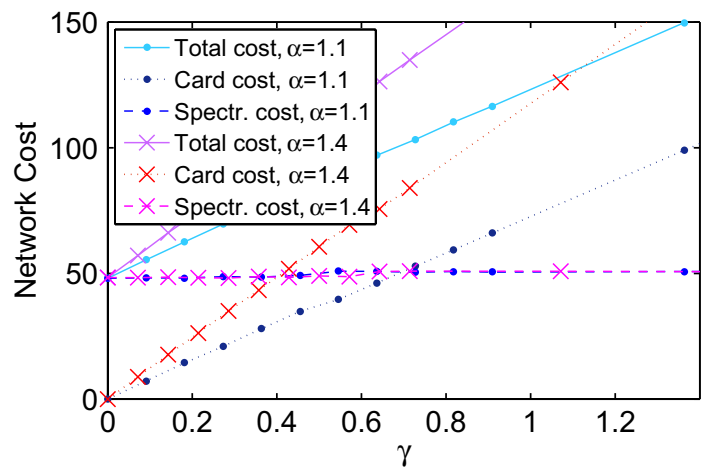

Fig. 6. Network cost versus $\gamma$ for $\alpha=1.1$ and $\alpha=1.4$ in a MMF/ SLR SSON ring for randomly generated traffic demands at $200 \mathrm{~Gb} / \mathrm{s}$.

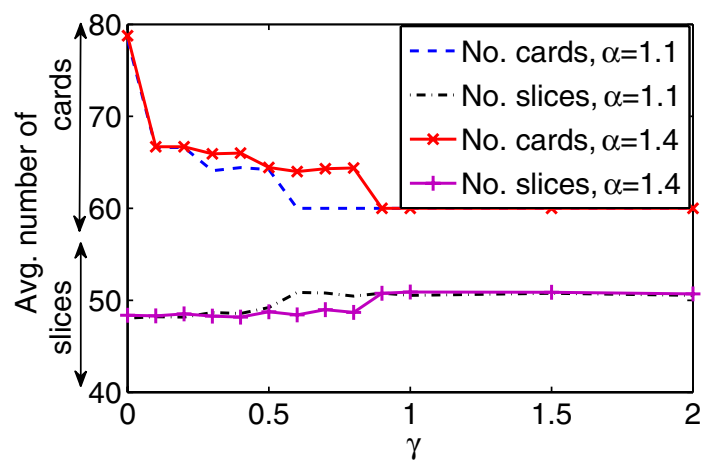

Fig. 7. Average number of cards/slots versus $\gamma$ in a MMF/SLR SSON ring for randomly generated traffic demands at $200 \mathrm{~Gb} / \mathrm{s}$.

slices used in the network. For instance, the PM-QPSK modulation format requires two times fewer cards than PM-16QAM, but it requires more frequency slices ( 1 and 2 slices more for 100 and $200 \mathrm{~Gb} / \mathrm{s}$, respectively). When $\gamma$ is higher than 0.7 , the average number of transponder cards and frequency slices remains constant and independent of $\gamma$. This happens because the slice cost is lower than the card cost and thus influences less the objective function. Accordingly, in the optimal solution, the PM-QPSK modulation format is selected for all the lightpaths, as it requires fewer cards but is less spectrally efficient than PM-16QAM.

The impact of the line rate optimization on the network cost is assessed in Fig. 8, which shows the total network cost for MMF/SLR-SSON and MMF/MLR-SSON, when $\alpha=1$.4. In the latest case, the requested traffic rate of $200 \mathrm{~Gb} / \mathrm{s}$ can be supported by either a single lightpath at $200 \mathrm{~Gb} / \mathrm{s}$ or two lightpaths at $100 \mathrm{~Gb} / \mathrm{s}$, using a modulation format able to satisfy the QoT requirements. The figure illustrates the total cost of the network versus the ratio between the cost of the card and the cost of the frequency slice, $\gamma$. For $\gamma$ close to 0, MLR is slightly more spectrally efficient than SLR (about 8\% more efficient), as shown also in Fig. 5; thus the total cost is slightly lower. Indeed, for the lightpath lengths between 250 and $400 \mathrm{~km}$, it is more spectrally efficient to split them in two $100 \mathrm{~Gb} / \mathrm{s}$ PM-16QAM lightpaths, instead of using a $200 \mathrm{~Gb} / \mathrm{s}$ PM-QPSK lightpath. For higher values of $\gamma$, the card cost becomes predominant. Thus, it is always preferable to transmit the demand

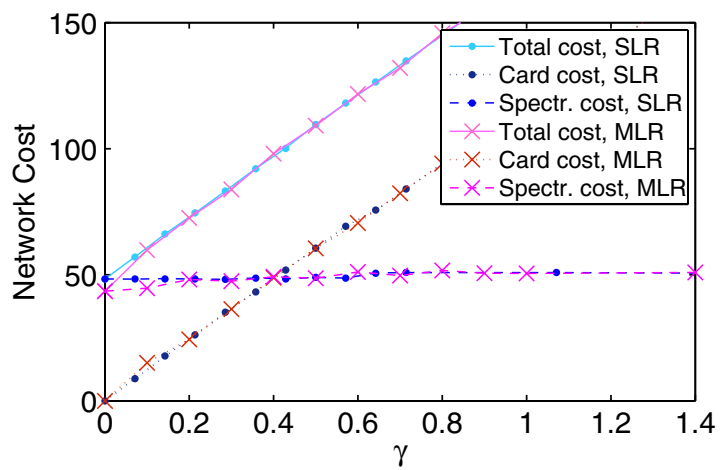

Fig. 8. Network cost versus $\gamma$, for $\alpha=1.4$ in MMF/SLR and MMF/ MLR rings for randomly generated traffic demands. 
traffic on a single lightpath, without exploiting the MLR features. As a result, the total cost for SLR and MLR becomes identical.

\section{Conclusions and Further Discussion}

This paper focused on the SA problem arising in SSON with a flexible grid. It proved that the SA problem can be mapped to the weighted coloring problem without the adjacency constraint and it is therefore NP-complete. Based on such a finding, an ILP formulation for the minimization of the total network cost, accounting for the cost of both the transponder cards and the frequency slice, was presented for a MMF/MLR-SSON in which the routing is fixed a priori.

Optimal results collected on a MMF/MLR-SSON ring show that spectrum efficiency should be balanced with the network card installation. The utilization of MLR transmission is especially beneficial when the spectrum efficiency is a concern. In particular, higher spectrum savings are achievable in small network sizes, when the more spectrally efficient modulation formats can be exploited for selected lightpaths (i.e., shorter than the maximum transparent reach). Spectrum saving already occurs at $100 \mathrm{~Gb} / \mathrm{s}$ when exploiting MMF and already justifies an early transition to MMF design in today's SSON network. The combination of MMF and MLR can further enhance the spectrum savings. Such spectrum saving can be up to $20 \%$ with respect to a SMF/SLR SSON network.

Although SA was assessed in a SSON ring, the results of this paper also find application in SSON with mesh topology, where the SA problem needs to be solved jointly with the lightpath routing problem, or RSA problem. Indeed, even in mesh networks, the adjacency constraint need not be enforced in the RSA problem, as proved in Corollary 1 presented in Appendix A. This enables a simplification of the ILP formulation and allows for resorting to already known algorithmic approaches for the routing and weighted coloring problems.

\section{Appendix A}

Corollary 1: In SSON with mesh topology, where the SA problem needs to be solved jointly with the lightpath routing problem, the spectrum continuity constraint need not be enforced in the joint optimization problem.

Proof: Optimally solving the joint RSA problem in a SSON with mesh topology is equivalent to selecting the best among all the possible routing solutions on which the SA problem has been optimally computed. For a given routing solution, Theorem 1 indicates that the SA problem reduces to a weighted coloring problem (i.e., the adjacency constraint need not be accounted for). Thus, also for the optimal solution of the joint optimization problem, the SA problem reduces to a weighted coloring problem. In conclusion, the joint RSA problem is equivalent to a joint routing and weighted coloring problem. On the optimal solution, frequency slice assignment can be performed by following the steps in the proof of Theorem 1.

\section{ACKNOWLEDGMENTS}

The work described in this paper was partially supported by the project "Architecture of Optical Networks and Nodes for High-Capacity Transmission and Access-Metro-Core Transport Based on Integrated Photonic Technologies (ARNO-T3)," funded by the Tuscany Region under the POR-CREO 2007-2013 program and by the FP-7 IDEALIST project under grant agreement no. 317999.

\section{REFERENCES}

[1] M. Jinno, B. Kozicki, H. Takara, A. Watanabe, Y. Sone, T. Tanaka, and A. Hirano, "Distance-adaptive spectrum resource allocation in spectrum-sliced elastic optical path network," IEEE Commun. Mag., vol. 48, no. 8, pp. 138-145, 2010.

[2] K. Christodoulopoulos, I. Tomkos, and E. Varvarigos, "Elastic bandwidth allocation in flexible OFDM-based optical networks," J. Lightwave Technol., vol. 29, no. 9, pp. 1354-1366, 2011.

[3] F. Cugini, G. Meloni, F. Paolucci, N. Sambo, M. Secondini, L. Gerardi, L. Pot, and P. Castoldi, "Demonstration of flexible optical network based on path computation element," J. Lightwave Technol., vol. 30, no. 5, pp. 727-733, Mar. 2012.

[4] D. Geisler, R. Proietti, Y. Yin, R. Scott, X. Cai, N. Fontaine, L. Paraschis, O. Gerstel, and S. Yoo, "The first testbed demonstration of a flexible bandwidth network with a real-time adaptive control plane," in Proc. of ECOC, Sept. 2011, pp. 1-3.

[5] N. Sambo, P. Castoldi, F. Cugini, G. Bottari, and P. Iovanna, "Toward high-rate and flexible optical networks," IEEE Commun. Mag., vol. 50, no. 5, pp. 66-72, May 2012.

[6] R. Casellas, R. Munoz, J. M. Fabrega, M. S. Moreolo, R. Martinez, L. Liu, T. Tsuritani, and I. Morita, "Design and experimental validation of a GMPLS/PCE control plane for elastic CO-OFDM optical networks," IEEE J. Sel. Areas Commun., vol. 31, no. 1, pp. 49-61, Jan. 2013.

[7] "Draft revised g.694.1 version 1.3," Unpublished ITU-T Study Group 15, Question 6.

[8] A. Patel, P. Ji, J. Jue, and T. Wang, "Routing, wavelength assignment, and spectrum allocation algorithms in transparent flexible optical WDM networks," Opt. Switching Networking, vol. 9, no. 3, pp. 191-204, 2012.

[9] L. Velasco, M. Klinkowski, M. Ruiz, and J. Comellas, "Modeling the routing and spectrum allocation problem for flexgrid optical networks," Photonic Network Commun., vol. 24, no. 3, pp. 177-186, 2012.

[10] M. Klinkowski and K. Walkowiak, "Routing and spectrum assignment in spectrum sliced elastic optical path network," IEEE Commun. Lett., vol. 15, no. 8, pp. 884-886, 2011.

[11] Y. Wang, X. Cao, Q. Hu, and Y. Pan, "Towards elastic and finegranular bandwidth allocation in spectrum-sliced optical networks," J.Opt. Commun. Netw., vol.4, no.11,pp.906-917,2012.

[12] A. Eira, J. Pedro, and J. Pires, "Cost-optimized dimensioning of translucent WDM networks with mixed-line-rate spectrumflexible channels," in 13th IEEE Int. Conf. on High Performance Switching and Routing (HPSR), 2012, pp. 185-190.

[13] O. Pedrola, A. Castro, L. Velasco, M. Ruiz, J. FernándezPalacios, and D. Careglio, "CAPEX study for a multilayer 
IP/MPLS-over-flexgrid optical network," J. Opt. Commun. Netw., vol. 4, no. 8, pp. 639-650, 2012.

[14] C. Rottondi, M. Tornatore, A. Pattavina, and G. Gavioli, "Routing, modulation level, and spectrum assignment in optical metro ring networks using elastic transceivers," J. Opt. Commun. Netw., vol. 5, no. 4, pp. 305-315, 2013.

[15] P. Ghobril, C. Zaiter, and E. Le Rouzic, "Rearrangement: From wavelength routed to sliced-spectrum optical networks," in Int. Conf. on Transparent Optical Networks (ICTON), 2012, pp. $1-4$.

[16] A. Nag, M. Tornatore, and B. Mukherjee, "Optical network design with mixed line rates and multiple modulation formats," J. Lightwave Technol., vol. 28, no. 4, pp. 466-475, 2010.

[17] P. Hansen, M. Labbé, and D. Schindl, "Set covering and packing formulations of graph coloring: Algorithms and first polyhedral results," Discrete Optim., vol. 6, no. 2, pp. 135-147, 2009.

[18] I. Bomze, M. Budinich, P. Pardalos, and M. Pelillo, "The maximum clique problem," in Handbook of Combinatorial Optimization, vol. 4. Kluwer Academic, 1999, pp. 1-74.

[19] P. Coll, J. Marenco, I. M. Diaz, and P. Zabala, "Facets of the graph coloring polytope," Ann. Operat. Res., vol. 116, no. 1, pp. 79-90, 2002.

[20] H. Zang, J. Jue, and B. Mukherjee, "A review of routing and wavelength assignment approaches for wavelength-routed optical WDM networks," Opt. Networks Mag., vol. 1, no. 1, pp. 47-60, 2000.

[21] M. Grötschel, L. Lovász, and A. Schrijver, "Relaxations of vertex packing," J. Comb. Theory, Ser. B, vol. 40, no. 3, pp. 330-343, 1986.

[22] M. Grotschel, L. Lovász, and A. Schrijver, Geometric Algorithms and Combinatorial Optimizations. Springer-Verlag, 1993.

[23] E. Lach and W. Idler, "Modulation formats for 100G and beyond," Opt. Fiber Technol., vol. 17, no. 5, pp. 377-386, 2011.

[24] A. Klekamp, R. Dischler, and F. Buchali, "Limits of spectral efficiency and transmission reach of optical-OFDM superchannels for adaptive networks," IEEE Photon. Technol. Lett., vol. 23 , no. 20 , pp. 1526-1528, 2011.

[25] B. T. Teipen, H. Griesser, and M. H. Eiselt, "Flexible bandwidth and bit-rate programmability in future optical networks," in Int. Conf. on Transparent Optical Networks (ICTON), 2012, pp. 1-4.

[26] Y.-K. Huang, E. Ip, T. Xia, G. Wellbrock, M.-F. Huang, Y. Aono, T. Tajima, and M. Cvijetic, "Mixed line-rate transmission $(112-\mathrm{Gb} / \mathrm{s}, 450-\mathrm{Gb} / \mathrm{s}$, and $1.15-\mathrm{Tb} / \mathrm{s})$ over $3560 \mathrm{~km}$ of fieldinstalled fiber with filterless coherent receiver," J. Lightwave Technol., vol. 30, no. 4, pp. 609-617, 2012.

[27] F. Cugini, F. Paolucci, N. Sambo, L. Poti, A. D'Errico, and G. Bottari, "Reliable flexible-ROADM architecture enabling modulation format adaptation," in Proc. European Conf. and Exhibition on Optical Communication (ECOC), 2012, pp. 1-3.

[28] "AMPL: A modeling language for mathematical programming," Dec. 2012 [Online]. Available: http://www.ampl.com/.

[29] "IBM ILOG CPLEX: High-performance mathematical programming engine," Dec. 2012 [Online]. Available: http:// www.ibm.com/.

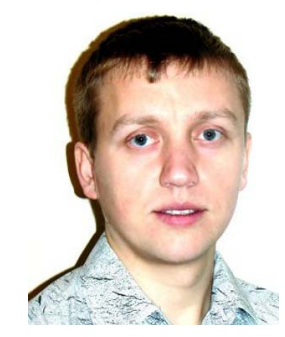

Ion Popescu received a Master's degree in communication networks engineering from the Scuola Superiore Sant'Anna, Pisa, Italy, in 2012 . He is currently a Ph.D. student in the Optics Department of Telecom Bretagne, Brest, France. His research interest is optical communication networks.

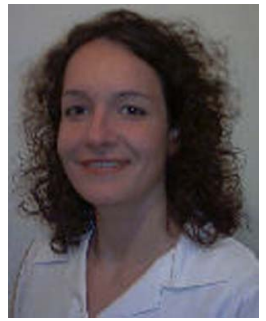

Isabella Cerutti holds a Ph.D. and a Laurea degree in electrical engineering from the University of Texas at Dallas (2002) and from the Politecnico di Torino, Italy (1998), respectively. From 2002 to 2006 , she was a post-doc research associate at the University of Texas at Dallas and then at Scuola Superiore Sant'Anna, Italy. She is currently an Assistant Professor at Scuola Superiore Sant'Anna. Her research interests include performance evaluation and design of optical and wireless networks, with special emphasis on energy efficiency.

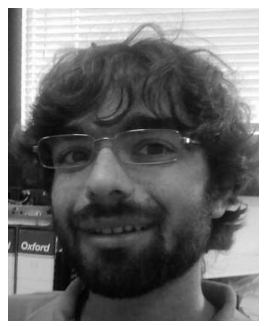

Nicola Sambo received a Laurea degree in telecommunications engineering from the University of Pisa, Italy, in 2004 and his Ph.D. degree from Scuola Superiore Sant'Anna, Pisa, Italy, in 2009. Currently he is an Assistant professor at Scuola Superiore Sant'Anna. He is also collaborating with CNIT, Pisa, Italy, and Ericsson, Pisa, Italy. His research interests include WDM network planning and control plane, transmission modeling, coherent transmission, GMPLS protocol suite, and path computation element.

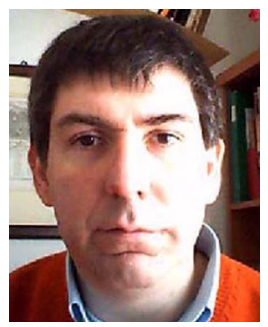

Piero Castoldi (Ph.D. degree in information technology) has been an Associate Professor at Scuola Superiore Sant'Anna, Pisa, Italy, since 2001. He spent abroad at Princeton University overall about two years in 1996, 1997, 1999, and 2000. In 2001, he visited the University of Texas at Dallas for two months. He has also served as Project Manager of many projects of the Inter-universitary National Consortium for Telecommunications (CNIT), and he was Director of the CNIT National Laboratory of Photonic Networks during the period of 2005-2010. He is also currently the Leader of the "Networks and Services" research area at the Institute of Communication, Information and Perception Technologies at Scuola Superiore Sant'Anna. His research interests cover reliability, switching paradigms, and control of optical networks, including application-network cooperation mechanisms, for smart grids and clouds. He is an IEEE Senior Member and he is the author of more than 200 publications in international journals and conference proceedings. 